\title{
Huiles et beurres de pulpes de fruits : revue des principales sources exploitées, teneurs en insaponifiables, propriétés et usages traditionnels d'intérêt cosmétique
}

\author{
Didier FONTANEL \\ ARDEX Sarl, 31 rue des frères Lumiere \\ 77100 Meaux ; Universités d'angers, \\ de Rennes- 1 et de Tours \\ $<$ ardex49@bbox.fr > \\ $<$ d.fontanel@gmail.com>
}

\begin{abstract}
Pulpy fruit oils and fats: Review of the main sources, unsaponifiable contents, properties and traditional uses related to interest in cosmetics Approximately fifteen pulpy fruits are widely used as sources of fats. Most of these fruits are provided from plants belong to the family Arecaceae. Fats from pulps surrounding seeds have composition including various unsaponifiable matters often including significant quantities of carotenoids. Others chemical families (e.g. tocopherols) are sometime in interesting contents among unsaponifiable material. Physiological effects and the traditional uses in topical applications of these oils are reported.
\end{abstract}

Key words: fats, vegetable oils, unsaponifiable matter, contents, fruit pulp, mesocarp, traditional use, cosmetics

D'un point de vue taxinomique, les pulpes des fruits exploitées pour leur richesse en lipides proviennent très majoritairement de palmiers (famille des Arecaceae) (tableau 1).

\section{Répartition géographique de ces espèces oléagineuses}

Neuf espèces oléagineuses citées sont présentes en Amazonie et s'avèrent donc des plantes de climats chauds et pluvieux. Trois espèces sont communes en Afrique centrale, tandis que trois autres sont cultivées sous des climats plus tempérés (tableau 2).

\section{Importance commerciale de ces matières grasses issues de pulpes}

Le commerce de ces matières grasses est très inégal. L'huile de palme est la plus produite au monde avec environ 48 millions de tonnes en 2010, tandis que I'huile d'olive se situe en $9^{\mathrm{e}}$ position, avec 3 millions de tonnes produites en 2010 (USDA, 2011). La place de I'huile d'avocat est bien plus modeste avec une estimation de production annuelle d'environ 1500 tonnes (Com. pers.). L'huile de pataua fit l'objet d'un commerce notoire au milieu du $X X X^{e}$ siècle. Les quantités exportées par le Brésil vers 1951 étaient de l'ordre de 200 tonnes/an (Janick et Paull, 2008).

En ce qui concerne les autres matières grasses, les volumes produits demeurent inférieures et difficiles à estimer. Les productions de graisses de pulpes d'aiélé, de palmier pêche et de safoutier, sont encore à la fois très limitées, artisanales, et surtout destinées à une consommation locale. II est utile d'ajouter que la majorité de ces matières grasses issues de mésocarpes sont à l'état figé ou semi-figé vers $15{ }^{\circ} \mathrm{C}$ (tableau 3).

\section{Teneurs en lipides et en matières insaponifiables}

Les teneurs en lipides dans les pulpes fraîches ou sèches ainsi que les teneurs en

Pour citer cet article : Fontanel D. Huiles et beurres de pulpes de fruits : revue des principales sources exploitées, teneurs en insaponifiables, propriétés et usages traditionnels d'intérêt cosmétique. OCL $2012 ; 19(4)$ : 232-237. doi : 10.1684/ocl.2012.0442 
Tableau 1. Espèces botaniques dont les matières grasses de pulpes de fruits sont les plus exploitées.

\begin{tabular}{|llll|}
\hline Noms usuels en France & Noms anglais & Nom botanique officiel & Famille botanique \\
\hline Aïélé, Elémier d'Afrique & Aiele, African canarium & Canarium schweinfurthii Engl. & Burseraceae \\
\hline Argousier & Seabukthorn & $\begin{array}{c}\text { Hippophae rhamnoides } \\
\text { L. subsp. Rhamnoïdes }\end{array}$ & Elaeagnaceae \\
\hline Avocat & Avocado & Persea americana Mill. & Lauraceae \\
\hline Awara & Tucum palm & Astrocaryum vulgare Mart. & Arecaceae \\
\hline Buriti & Aguaje & Mauritia flexuosa L. f. & Arecaceae \\
\hline Comou & Turu palm & Oenocarpus bacaba Mart. & Arecaceae \\
\hline Maripa & Maripa palm & Attalea maripa (Aubl.) Mart. & Arecaceae \\
\hline Olivier & Olive & Olea europaea L. & Oleaceae \\
\hline Palmier à huile & African oil palm & Elaeis guineensis Jacq. & Arecaceae \\
\hline Palmier à huile d'Amérique & American oil palm & Elaeis oleifera (Kunth) Cortés & Arecaceae \\
\hline Palmier pêche & Peach palm & Bactris gasipaes Kunth & Arecaceae \\
\hline Pataua & Pataua palm & Oenocarpus bataua Mart. var. bataua & Arecaceae \\
\hline Pequi & Pequi & Caryocar brasiliense Cambess. & Caryocaraceae \\
\hline Pequia & Pekea nut & Caryocar villosum (Aubl.) Pers. & Caryocaraceae \\
\hline Safoutier & African pear & Dacryodes edulis (G. Don) Lam. & Burseraceae \\
\hline
\end{tabular}

* Caryocar coriaceum Wittm. : aussi baptisé Pequi. Selon les avis, il s'agit d'une espèce à part entière ou bien non valide.

matières insaponifiables sont reportées dans le tableau 3. Les teneurs en matières insaponifiables de ces matières grasses, déterminées par extraction au solvant, sont assez homogènes et courantes $(0,4$ 2,2\%), à l'exception de l'huile d'avocat. Pour cette dernière, les teneurs habituellement rapportées sont comprises entre 1,4 et 3,0 \% de matières insaponifiables dans I'huile de la pulpe fraîche ou sèche. Néanmoins, des teneurs bien plus élevées ont été signalées: de 4,8$12,2 \%$ dans de I'huile issue de la pulpe fraîche (Gutfinger et Letan, 1974) et de 4,0-8,8 \% dans I'huile de pulpe sèche (Lozano et al., 1993).

Tableau 2. Répartition géographiques des espèces oléagineuses exploitées pour la richesse en matiere grasse de la pulpe du fruit.

\begin{tabular}{|ll|}
\hline Répartition des espèces & Noms français \\
\hline Amazonie & $\begin{array}{l}\text { Awara, Buriti, Comou, Maripa, } \\
\text { Pataua, Pequi, Pequia }\end{array}$ \\
\hline De I'Amérique centrale au Brésil & Palmier à huile d'Amérique \\
\hline Amérique centrale et du Sud & Palmier pêche \\
\hline Diverses régions tropicales humides & Palmier à huile d'Afrique \\
\hline Du Nigeria à l'Angola ; vers l'est jusqu'à l'Ouganda & Safoutier \\
\hline Afrique & Aïélé \\
\hline Diverses régions tropicales et méditerranéennes & Avocatier \\
\hline Surtout bassin méditerranéen & Olivier \\
\hline Europe, Canada, Russie, Inde... & Argousier \\
\hline
\end{tabular}

couleur orangée due à la présence de caroténoïdes dont les teneurs connues les plus remarquables varient de 38 à 528 $\mathrm{mg} / 100 \mathrm{~g}$ de matière grasse. De telles teneurs sont les plus élevées parmi les corps gras végétaux exploités.

D'après la littérature actuelle, les teneurs en tocophérols les plus remarquables parmi ces matières grasses de pulpes, sont celles de l'argousier et du pataua (tableau 4).

Le squalène est en quantités importantes bien connues dans I'huile d'olive (90-870 mg/100 g).

Quant à la fraction insaponifiable de I'huile d'avocat, elle contient des alcools gras insaturés dont les proportions n'ont pas encore été mentionnées dans la littérature.

\section{Effets physiologiques d'intérêt cosmétiques et usages traditionnels de ces matières grasses de pulpes de fruits}

Les propriétés physiologiques et usages traditionnels d'intérêt cosmétique de ces matières grasses de pulpes ont été résumés dans le tableau 5. La plupart sont appliquées sur la peau pour préserver le film hydrolipidique et ainsi 
Tableau 3. Teneurs en lipides de pulpe de fruits et en matieres insaponifiables de la matiere grasse.

\begin{tabular}{|c|c|c|c|c|}
\hline $\begin{array}{l}\text { Végétal } \\
\text { (consistance de la } \\
\text { matière grasse à } \\
\left.15^{\circ} \mathrm{C}\right)\end{array}$ & $\begin{array}{l}\text { Teneur en lipides } \\
\text { dans la pulpe } \\
\text { fraîche }(\mathbf{g} / 100 \mathrm{~g})\end{array}$ & $\begin{array}{l}\text { Teneur en lipides } \\
\text { dans la pulpe } \\
\text { sèche }(\mathrm{g} / 100 \mathrm{~g})\end{array}$ & $\begin{array}{l}\text { Teneurs en matières } \\
\text { insaponifiables } \\
\text { de la matière grasse } \\
(\mathrm{g} / 100 \mathrm{~g})\end{array}$ & Sources \\
\hline $\begin{array}{l}\text { Aïélé } \\
\text { (beurre) }\end{array}$ & & $36-40$ & $\begin{array}{l}1,0-1,3 \\
\text { (méthodes imprécise } \\
\text { ou particulière) }\end{array}$ & $\begin{array}{l}\text { Agbo N'zi et al., } 1992 ; \\
\text { Kapseu et Parmentier, } 1997\end{array}$ \\
\hline $\begin{array}{l}\text { Argousier } \\
\text { (huile) }\end{array}$ & & $11,6-24,3$ & (non publiée) & Yang et Kallio, 2001 \\
\hline \multirow[t]{2}{*}{$\begin{array}{l}\text { Avocat } \\
\text { (huile) }\end{array}$} & $8-22$ & & $\begin{array}{l}1,4-12,2 \\
\text { (extr. éthoxyéthane) }\end{array}$ & \multirow{2}{*}{4 publications (Fontanel, 2011) } \\
\hline & & $65-66$ & $\begin{array}{l}1,7-8,8 \\
\text { (extr. éthoxyéthane) }\end{array}$ & \\
\hline \multirow[t]{3}{*}{$\begin{array}{l}\text { Awara } \\
\text { (beurre) }\end{array}$} & & 18,2 & $\begin{array}{l}2,2 \\
\text { (extr. éthoxyéthane) }\end{array}$ & Mambrim et Barrera-Arellano, 1997 \\
\hline & & 16,5 & $\begin{array}{l}0,3 \\
\text { (extr. n-hexane) }\end{array}$ & Bereau, 2001 \\
\hline & 9,5 & & $\begin{array}{l}1,0 \\
\text { (méthode imprécise) } \\
0,75 \\
\text { (extr. N-hexane) }\end{array}$ & $\begin{array}{l}\text { Lubrano et Robin, } 1997 \\
\text { Bony et al., } 2012\end{array}$ \\
\hline $\begin{array}{l}\text { Buriti } \\
\text { (huile) }\end{array}$ & & 22 & $\begin{array}{l}0,5-0,9 \\
\text { (méthode imprécise) }\end{array}$ & Lognay et al., 1987 \\
\hline \multirow[t]{2}{*}{$\begin{array}{l}\text { Comou } \\
\text { (semi-figée) }\end{array}$} & 14 & & $\begin{array}{l}0,7 \\
\text { (méthode imprécise) }\end{array}$ & Lubrano et Robin, 1997 \\
\hline & & 29 & $\begin{array}{l}0,2 \\
\text { (extr. n-hexane) }\end{array}$ & Bereau, 2001 \\
\hline $\begin{array}{l}\text { Maripa } \\
\text { (beurre) }\end{array}$ & & 13,6 & $\begin{array}{l}1,0 \\
\text { (méthode n-hexane) }\end{array}$ & Bereau, 2001 \\
\hline $\begin{array}{l}\text { Olivier } \\
\text { (huile) }\end{array}$ & $13-15$ & & $\begin{array}{l}0,8-1,5 \\
\text { (ext. éthoxyéthane) }\end{array}$ & $\begin{array}{l}\text { Gutfinger et Letan, } 1974 \text {; } \\
\text { Souci et al., } 2000\end{array}$ \\
\hline $\begin{array}{l}\text { Palmier à huile } \\
\text { (beurre) }\end{array}$ & & 46,5 & $\begin{array}{l}0,4-1,2 \\
\text { (ext. éthoxyéthane) }\end{array}$ & $\begin{array}{l}\text { (Fedeli et al., } 1966 ; \\
\text { Codex alimentarius, } 2009 ; \\
\text { Kapseu et Parmentier, } 1997\end{array}$ \\
\hline \multirow{2}{*}{$\begin{array}{l}\text { Palmier à huile } \\
\text { d'Amérique }^{\prime} \\
\text { (beurre) }\end{array}$} & 30 & & $\begin{array}{l}0,5-1,0 \\
\text { (méthode non précisée) }\end{array}$ & Mensier, 1957 \\
\hline & & 13,3 & $\begin{array}{l}1,4 \\
\text { (extr. n-hexane) }\end{array}$ & Bereau, 2001 \\
\hline $\begin{array}{l}\text { Palmier pêche } \\
\text { (beurre ou semi-figée) }\end{array}$ & & 31,2 & $\begin{array}{l}0,4 \\
\text { (extr. n-hexane) }\end{array}$ & Bereau, 2001 \\
\hline $\begin{array}{l}\text { Pataua } \\
\text { (huile) }\end{array}$ & & $23-52$ & $\begin{array}{l}0,8-1,0 \\
\text { (extr. éthoxyéthane) } \\
1,3 \\
\text { (extr. n-hexane) }\end{array}$ & $\begin{array}{l}\text { Gomes Da Silva et Fedeli, 1995; } \\
\text { Mambrim et Barrera-Arellano, } 1997 \text {; } \\
\text { Montúfar et al., } 2010 \\
\text { Bereau, } 2001\end{array}$ \\
\hline $\begin{array}{l}\text { Pequi } \\
\text { (beurre) }\end{array}$ & & 70,1 & (non publiée) & $\begin{array}{l}\text { Gomez de Brito Mariano et al., } \\
2009\end{array}$ \\
\hline $\begin{array}{l}\text { Pequia } \\
\text { (beurre) }\end{array}$ & & 64,5 & $\begin{array}{l}>0,6 \\
\text { (méthode par CPG) }\end{array}$ & Marx et al., 1997 \\
\hline $\begin{array}{l}\text { Safoutier } \\
\text { (semi-figée) }\end{array}$ & & $45-57$ & $\begin{array}{l}0,9-2,3 \\
\text { (extr. éthoxyéthane } \\
\text { et méthode particulière) }\end{array}$ & $\begin{array}{l}\text { Ucciani et Busson, } 1963 \text {; } \\
\text { Kapseu et Parmentier, } 1997\end{array}$ \\
\hline
\end{tabular}

extr. : extraction après saponification par ... ; CPG : chromatographie en phase gazeuse 
Tableau 4. Teneurs inhabituelles en familles chimiques ou constituants insaponifiables dans ces matieres grasses issues de pulpes.

\begin{tabular}{|c|c|c|}
\hline Végétal & $\begin{array}{l}\text { Teneurs inhabituelles en familles chimiques } \\
\text { ou constituants insaponifiables dans } \\
\text { la matière grasse issue de la pulpe } \\
(\text { en } \mathrm{mg} / 100 \mathrm{~g})\end{array}$ & Sources \\
\hline Aiélé & Encore peu connues, beurre de couleur verte & \\
\hline \multirow[t]{2}{*}{ Argousier } & $\begin{array}{l}\text { Caroténoïdes : } 242 \\
\text { (méthode par spectrophotométrie) }\end{array}$ & $\begin{array}{l}\text { Arimboor et al., } 2006 \text {; } \\
\text { Cenkowski et al., } 2006\end{array}$ \\
\hline & $\begin{array}{l}\text { Tocophérols : } 141-471 \\
\text { (méthode par CLHP) }\end{array}$ & $\begin{array}{l}\text { Arimboor et al., } 2006 \text {; } \\
\text { Cenkowski et al., } 2006\end{array}$ \\
\hline Avocat & $\begin{array}{l}\text { Fraction polaire (1,2,4-trihydroxy-n-heptadec-16-ène } \\
\text { majoritaire) : } 544 \\
\text { (méthode par CCM) }\end{array}$ & Itoh et al., 1975 \\
\hline \multirow[t]{2}{*}{ Awara } & $\begin{array}{l}\text { Caroténoïdes : } 245-528 \\
\text { (méthode par spectrophotométrie) }\end{array}$ & $\begin{array}{l}\text { Mambrim et Barrera-Arellano, } \\
1997\end{array}$ \\
\hline & $\begin{array}{l}\text { Caroténoïdes : } 164 \text { (exprimées en } \beta \text {-carotène) } \\
\text { (méthode par CLHP) }\end{array}$ & Bony et al., 2012 \\
\hline Buriti & $\begin{array}{l}\text { Caroténoïdes : 90-173 } \\
\text { (méthodes par CLHP et spectrophotométrie) }\end{array}$ & Lognay et al., 1987 \\
\hline Comou & Encore peu connues, matière grasse de couleur verte & \\
\hline Maripa & $\left({ }^{*}\right)$, beurre de couleur orange & \\
\hline Olivier & $\begin{array}{l}\text { Squalène : } 90-870 \\
\text { (méthodes par CPG et CLHP) }\end{array}$ & $\begin{array}{l}\text { Gutfinger et Letan, } 1974 \text {; } \\
\text { De Leonardis et al., 1998; } \\
\text { Nenadis et Tsimidou, 2002) }\end{array}$ \\
\hline Palmier à huile & $\begin{array}{l}\text { Caroténoïdes : } 38-200 \\
\text { (méthode par spectrophotométrie) }\end{array}$ & $\begin{array}{l}\text { Codex alimentarius, } 2009 \text {; } \\
\text { Yap et al., } 1991\end{array}$ \\
\hline Palmier à huile d'Amérique & $\begin{array}{l}\text { Caroténoïdes : } 435 \\
\text { (méthode par spectrophotométrie) }\end{array}$ & Yap et al., 1991 \\
\hline Palmier pêche & $\left({ }^{*}\right)$, matière grasse de couleur orange & \\
\hline Pataua & $\begin{array}{l}\text { Tocophérols : } 197\left(^{* *}\right) \\
\text { (méthode par CLHP) }\end{array}$ & Montúfar et al., 2010 \\
\hline Pequi & $\begin{array}{l}\text { Caroténoïdes : teneurs très variables, à confirmer, } \\
\text { beurre de couleur jaune }\end{array}$ & \\
\hline Pequia & $\left(^{*}\right)$, beurre de couleur jaune & \\
\hline Safoutier & Encore peu connues, matière grasse de couleur jaune à vert & \\
\hline
\end{tabular}

$\left.{ }^{*}\right)$ : Pas de teneurs inhabituelles parmi les familles ou constituants insaponifiables selon les connaissances actuelles.

CCM : chromatographie couche mince ; CLHP : chromatographie liquide haute performance ; CPG : chromatographie en phase gazeuse.

$\left.{ }^{(*}\right)$ : Des teneurs très différentes $(9-11 \mathrm{mg} / 100 \mathrm{~g})$, bien plus banales, ont aussi été publées (Bereau, 2001).

freiner la déshydratation. Deux d'entre elles, I'huile de pulpe du fruit d'argousier et le beurre de pequi, ont présenté des effets cicatrisants lors d'essais par applications topiques (Wang et al., 2006 ; De Oliveira et al., 2010 ; Saraiva et al., 2010).

Quelques-unes de ces matières grasses sont, par ailleurs, parfois utilisées en alimentation et presque toutes sont comestibles.

Un possible intérêt cosmétique commun aux matières grasses de pulpes de fruits est parfois avancé depuis peu. Elles seraient "hypoallergéniques » (Internet 2, 2011). Jusqu'à présent, la littérature ne semble pas contenir d'argument scientifique à ce sujet. Par contre, quelques critiques s'élèvent parfois concernant l'emploi en cosmétique de certaines matières grasses issues de graines ou d'amandes. Ainsi, depuis plusieurs années, un nombre grandissant de médecins déconseillent l'application $d^{\prime}$ huiles de graines ou amandes en particulier I'huile d'amande douce sur la peau des nourrissons. Pour cela, ils s'appuient sur le fait que ces fruits à écale sont la cause par voie orale d'allergies parmi les populations de tout âge des pays développés (Moneret-Vautrin, 2008). Il est bien connu que les protéines de réserve des graines oléagineuses sont principalement responsables des causes d'allergies liées à la consommation de ces graines. Des analyses récentes ont mis en évidence la présence de protéines issues de graines oléagineuses dans des huiles brutes, et aussi des traces dans des huiles raffinées. Néanmoins, si des cas rapportés d'allergies aux huiles végétales issues de graines oléagineuses (amande, arachide...) lors d'applications topiques font débat, ils restent encore assez marginaux (Ring et Möhrenschlager, 2007 ; Guillet et Guillet, 2000). II serait intéressant que des essais de tolérance soient menés chez des sujets aux peaux réactives afin de comparer l'incidence d’huiles végétales issues de pulpes et 
Tableau 5. Propriétés d'intérêt cosmétique, usages traditionnels par voie topique et comestibilité de ces matieres grasses issues de pulpes.

\begin{tabular}{|c|c|}
\hline Huiles de pulpes & Propriétés d'intérêt cosmétique et usages traditionnels \\
\hline Aiélé & - Usage alimentaire de I'huile dans plusieurs terroirs d'Afrique. \\
\hline \multirow[t]{3}{*}{ Argousier } & - Huile activant la cicatrisation de brûlures cutanées (Wang et al., 2006). \\
\hline & $\begin{array}{l}\text { - En Russie et en Chine, cette huile est utilisée par voie topique pour traiter les brûlures } \\
\text { de la peau (Wang et al., 2006). }\end{array}$ \\
\hline & - Employée en cosmétique pour sa richesse en caroténoïdes et en tocophérols. \\
\hline \multirow[t]{2}{*}{ Avocat } & - Huile alimentaire. \\
\hline & - En cosmétique, huile réputée régénératrice et pour maintenir l'hydratation de la peau. \\
\hline \multirow[t]{3}{*}{ Awara } & - Propriétés anti-inflammatoires in vivo de I’huile chez des rongeurs (Bony et al., 2012). \\
\hline & $\begin{array}{l}\text { - Utilisée en cosmétique au Brésil en cas de peau déshydratée, dans des lotions pour le corps, } \\
\text { et des produits pour cheveux abîmés (Internet 1, 2011). }\end{array}$ \\
\hline & - Beurre consommé en Amazonie (Bereau, 2001). \\
\hline \multirow[t]{3}{*}{ Buriti } & - Huile testée à titre d’adjuvant dans une formule filtre solaire (Zanatta et al., 2010). \\
\hline & $\begin{array}{l}\text { - Au Brésil, l'huile est utilisée par l'industrie cosmétique comme filtre solaire et pour réduire } \\
\text { le dessèchement de la peau. Une formule " anti-âge » de cette huile augmente l'élasticité } \\
\text { de la peau (Internet 1, 2011). }\end{array}$ \\
\hline & - Fonction en cosmétique : « skin conditionning» (INCI Directory). \\
\hline Comou & - Fonction en cosmétique : émollient, hydratant (INCI Directory). \\
\hline \multirow[t]{2}{*}{ Maripa } & - Fonction en cosmétique : « skin conditionning» (INCI Directory). \\
\hline & - Beurre comestible (Bereau, 2001). \\
\hline Olivier & $\begin{array}{l}\text { - Huile d'usages cosmétique et alimentaire très répandus. Stabilité supérieure à la plupart } \\
\text { des autres huiles végétales. }\end{array}$ \\
\hline Palmier à huile (d'Afrique) & - Beurre alimentaire et d’usage cosmétique répandu. \\
\hline Palmier à huile d'Amérique & - Beurre utilisée comme substitut du beurre laitier (Balick, 1979). \\
\hline Palmier pêche & $\begin{array}{l}\text { - Peu d'informations sur l'emploi de l'huile. La pulpe du fruit est consommée après cuisson } \\
\text { dans I'eau salée (Bereau, 2001). }\end{array}$ \\
\hline \multirow[t]{3}{*}{ Pataua } & - Fonction en cosmétique : émollient, hydratant (INCI Directory). \\
\hline & $\begin{array}{l}\text { - Au Brésil, cette huile est utilisée comme soin vitalisant de la peau et des cheveux, et pour } \\
\text { le traitement des pellicules (Internet 1, 2011). }\end{array}$ \\
\hline & - Huile comestible (Bereau, 2001). \\
\hline \multirow[t]{3}{*}{ Pequi } & $\begin{array}{l}\text { - Propriétés anti-inflammatoires topiques et cicatrisantes du beurre chez le rongeur } \\
\text { (De Oliveira et al., } 2010 \text {; Saraiva et al., 2010). }\end{array}$ \\
\hline & - Fonction en cosmétique : "skin conditionning » (INCI Directory) \\
\hline & - Beurre alimentaire au Brésil (Mensier, 1957). \\
\hline \multirow[t]{2}{*}{ Pequia } & - Fonction en cosmétique : émollient (INCI Directory) \\
\hline & - Beurre alimentaire (Mensier, 1957) \\
\hline Safoutier & - Usage alimentaire de I'huile dans plusieurs régions d'Afrique centrale. \\
\hline
\end{tabular}

$\mathrm{d}^{\prime}$ autres issus de graines. Toutefois jusqu'à présent, les allergies de contact imputées à des huiles de pulpes demeurent extrêmement rares.

\section{RÉFÉRENCES}

Agbo N'zi G, Chatigre KO, Simard RE. Canarium schweinfurthii Engl.: Chemical composition of the fruit pulp. / Am Oil Chem Soc $1992 ; 69$ : 317-20.

Arimboor R, Venugopalan VV, Sarinkumar K, Arumughan C, Sawhney RC. Integrated processing of fresh Indian sea buckthorn (Hippophae rhamnoides) berries and chemical evaluation of products. / Sci Food Agric 2006 ; 86 : 2345-53.

Balick MJ. Amazonian oil palms of promise: a survey. Econ Bot 1979 ; 33 : 11-28.

Bereau D. Huiles et fractions insaponifiables de huit espèces de palmiers amazoniens. Thèse de doctorat. Institut National Polytechnique de Toulouse, 2001.

Bony E, Boudard F, Brat P, et al. Awara (Astrocaryum vulgare M.) pulp oil: Chemical characterization, and anti-inflammatory properties in a mice model of endotoxic shock and a rat model of pulmonary inflammation. Fitoterapia 2012 ; 83 : 33-43.

Cenkowski S, Yakimishen R, Przybylski R, Muir WE. Quality of extracted sea buckthorn seed and pulp oil. Can Biosystems Eng 2006 ; 48 : 3.9-3.16.

Codex alimentarius. Norme pour les huiles végétales portant un nom spécifique. CodexStan 210-1999 (Adopté en 1999 ; révisions 2001, 2003, 2009 ; amendements 2005). In: Codex alimentarius, FAO/WHO, 2009, 8. 
De Leonardis A, Macciola V, De Felice M. Rapid determination of squalene in virgin olive oils using gas-liquid chromatography. Ital J Food Sci $1998 ; 10: 75-80$.

De Oliveira ML, Nunes-Pinheiro DC, Tomé $\mathrm{AR}$, et al. In vivo topical anti-inflammatory and wound healing activities of the fixed oil of Caryocar coriaceum Wittm. seeds. J Ethnopharmacol $2010 ; 129$ : 214-9.

Fedeli E, Lanzani A, Capella P, Jacini G. Triterpene alcohols and sterols of vegetable oils. J Am Oil Chem Soc 1966 ; 43 : 254-6.

Fontanel D. Huiles végétales. Teneurs en matieres insaponifiables. Tec \& Doc, Paris, 2011.

Gomes Da Silva W, Fedeli E. Pataua - A source of vegetable edible oil. New alternatives. Riv Ital Sost Grasse 1995 ; 72 : 303-6.

Gomez de Brito Mariano R, Couri S, Pereira Fretas $S$. Enzymatic technology to improve oil extraction from Caryocar brasiliense camb. (Pequi) pulp. Rev Bras Frutic 2009 ; $31: 637-43$.

Guillet G, Guillet MH. Sensibilisation alimentaire percutanée: A propos d'une sensibilisaton percutanée à l'amande chez le nourrisson et d'une étude de topiques chez 27 patients atteints d'allergie alimentaire. Allerg Immunol (Paris) 2000; 32 : 309-11.

Gutfinger T, Letan A. Studies of unsaponifiables in several vegetable oils. Lipids $1974 ; 9$ : 658-63.

$\mathrm{INCl}$ Directory - Function. http://www.specialchem4cosmetics.com/services/inci/index. aspx (Site consulté sur Internet le 21.11.2011).

Internet 1 : http://www.amazonoil.com. br/en/products/oils/tucuma_pulp.htm (Site consulté le 21.11.2011).

Internet 2 : http://sourcebio.blogspot.com/. (Site consulté le 21.11.2011).

Itoh T, Tamura T, Matsumoto T, Dupaigne P. Etudes sur I'huile d'avocat, en particulier sur la fraction stérolique de l'insaponifiable. Fruits $1975 ; 30$ : 687-95.

Janick J, Paull RE (eds). The encyclopedia of fruts and nuts. Wallingford, $\mathrm{GB}$ : $\mathrm{CAB}$ International, 2008.

Kapseu C, Parmentier M. Composition en acides gras de quelques huiles vegetales du Cameroun. Sci Aliments 1997; 17: 325-31.

Lognay G, Trevejo E, Jordan E, Marlier M, Severin M, Ortiz de Zarate I. Investigations on Mauritia flexuosa L. oil. Grasas Aceites 1987 ; 38 : 303-7.

Lozano YF, Mayer CD, Bannon C, Gaydou EM. Unsaponifiable matter, total sterol and tocopherol contents of avocado oil varieties. J Am Oil Chem Soc 1993 ; 70 : 561-5.

Lubrano C, Robin JR. Etude des Composees majeurs d'huiles de pulpe de fruits de six espèces de palmiers de Guyane. Acta Bot Gallica 1997 ; 144 : 497-9.

Mambrim MCT, Barrera-Arellano D. Characterization of palm tree fruit oils from Brazilian Amazonia region. Grasas Aceites 1997 ; 48 : 154-8.

Marx F, Andrade EHA, Maia JG. Chemical composition of the fruit pulp of Caryocar villosum. Z Lebensm Unters Forsch A 1997 ; 204 : 442-4.

Mensier P-H. Dictionnaire des huiles végétales. Paris : Paul Lechevalier, 1957.

Moneret-Vautrin DA. Épidémiologie de I'allergie alimentaire. Rev Fr d'Allergol Immunol Clin ; 2008 ; 48 : 171-8.

Montúfar R, Laffargue A, Pintaud JC, Hamon $S$, Avallone S, Dussert S. Oenocarpus bataua Mart. (Arecaceae): Rediscovering a source of high oleic vegetable oil from Amazonia. J Am Oils Chem Soc $2010 ; 87$ : 167-72.

Nenadis N, Tsimidou M. Determination of squalene in olive oil using fractional crystallization for sample preparation. I Am Oil Chem Soc $2002 ; 79$ : 257-9.
Ring J, Möhrenschlager M. Allergy to peanut oil - clinically relevant? I Eur Acad Dermatol Venereol 2007 ; 21 : 452-5.

Saraiva RA, Araruna MK, Oliveira RC, et al. Topical anti-inflammatory effect of Caryocar coriaceum Wittm. (Caryocaraceae) fruit pulp fixed oil on mice ear edema induced by different irritant agents. I Ethnopharmacol $2010 ; 136$ : 504-10.

Souci SW, Fachmann W, Kraut H. Food composition and nutrition table. Medipharm, Stuttgart ; USA: CRC Press Boca Raton, 2000. Ucciani E, Busson F. Contribution à l'étude des corps gras de Pachylobus edulis Don (Burseraceae). Oléagineux 1963 ; 18 : 253-5.

Ucciani E. Nouveau dictionnaire des huiles végétales. Paris: Tec \& Doc, 1994.

USDA (United States Department of Agriculture). Foreign Agricultural Service Table 03 : Major Vegetable Oils: World Supply and Distribution (Commodity View), December 9, 2011. http://www.fas.usda.gov/psdonline/psdreport.aspx?hidReportRetrievalName= BVS\&hidReportRetrievalID $=533 \&$ hidReport RetrievalTemplateID $=5$ (Site consulté le 03.01.2012).

Wang ZY, Luo XL, He CP. Management of burn wounds with Hippophae rhamnoides oil. Nan Fang Yi Ke Da Xue Xue Bao 2006 ; 26 : 124-5.

Yang B, Kallio HP. Fatty acid composition of lipids in sea buckthorn (Hippophae rhamnoides L.) berries of different origins. J Agric Food Chem $2001 ; 49$ : 1939-47.

Yap SC, Choo YM, Ooi CK, Ong ASH, Goh $\mathrm{SH}$. Quantitative analysis of carotenes in the oil from different palm species. Elaeis 1991 ; $3: 369-78$.

Zanatta CF, Mitjans M, Urgatondo V, RochaFilho PA, Vinardell MP. Photoprotective potential of emulsions formulated with Buriti oil (Mauritia flexuosa) against UV irradiation on keratinocytes and fibroblasts cell lines. Food Chem Toxicol 2010 ; 48 : 70-5. 